\title{
A Phase I, Dose-Escalation Trial of Pazopanib in Combination with Cisplatin in Patients with Advanced Solid Tumors: A UNICANCER Study
}

\author{
Véronique Diéras - Thomas Bachelot · Mario Campone · \\ Nicolas Isambert · Florence Joly • Christophe Le Tourneau • Philippe Cassier • \\ Emmanuelle Bompas · Pierre Fumoleau · Sabine Noal • \\ Christine Orsini · Marta Jimenez $\cdot$ Diane Charlotte Imbs · Etienne Chatelut
}

Received: May 25, 2016/ Published online: August 18, 2016

(C) The Author(s) 2016. This article is published with open access at Springerlink.com

\begin{abstract}
Introduction: To determine the feasibility, maximum-tolerated dose (MTD), and dose-limiting toxicities (DLT) of pazopanib in combination with cisplatin.
\end{abstract}

Methods: Patients with advanced malignancies were included in a $3+3$ dose-escalation phase I study. Pazopanib administration started 8 days before the first infusion of cisplatin; some patients were treated according to a reverse sequence (cisplatin first). Five dose levels (DLs)

Enhanced content To view enhanced content for this article go to http://www.medengine.com/Redeem/ 66E4F06070E18B18.

V. Diéras $(\bowtie) \cdot$ C. Le Tourneau

Department of Medical Oncology, Institut Curie,

Saint-Cloud, Paris, France

e-mail: veronique.dieras@curie.fr

T. Bachelot $\cdot$ P. Cassier

Department of Medical Oncology, Centre Léon

Bérard, Lyon, France

M. Campone · E. Bompas

Department of Medical Oncology, Institut de

Cancérologie de l'Ouest, Nantes, France

N. Isambert · P. Fumoleau

Department of Medical Oncology, Centre

Georges-François Leclerc, Dijon, France were planned. MTD was based on DLT observed during cycles 1 and 2 .

Results: Thirty-five patients were enrolled. The MTD was reached at the first DL, (pazopanib $400 \mathrm{mg}$ daily + cisplatin $75 \mathrm{mg} / \mathrm{m}^{2}$ every 21 days). Main DLTs were pulmonary embolism, neutropenia, thrombocytopenia, and elevation of liver enzymes. Overall, most common adverse events were anemia (83\%), fatigue (80\%), thrombocytopenia (80\%), neutropenia (73\%), hypertension (59\%), neurotoxicity (56\%), and anorexia (53\%). Sixteen patients (46\%) discontinued the study due to toxicity. One patient (sarcoma) had a complete response, and three patients (one with breast cancer and two with ovarian cancers) had

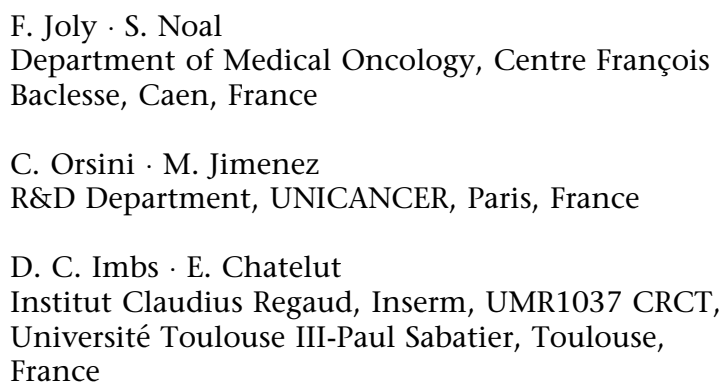


a partial response. Pharmacokinetic (PK) analyses showed interactions with aprepitant, resulting in increased exposure to pazopanib, which might explain partly the poor tolerance of the combination.

Conclusion: Cisplatin and pazopanib could not be administered at their single agent full doses, partly due to a PK interaction between pazopanib and aprepitant.

Funding: This work was funded by GlaxoSmithKline and by the charity Ligue Nationale de Lutte Contre le Cancer.

Trial registered: ClinicalTrials.gov identifier, NCT01165385.

Keywords: Aprepitant; Cisplatin; Pazopanib; Phase I trial; Safety; Solid tumors

\section{INTRODUCTION}

Therapeutic options are limited in advanced or refractory malignancies. The addition of angiogenesis inhibitors to cytotoxic drugs could enhance antitumor activity. Moreover, anti-angiogenic agents may normalize "leaky" tumor vasculature and increase the delivery of chemotherapy agents to the tumor site and enhance their efficacy [1]. The combination of chemotherapy and angiogenesis inhibition is already approved in some tumor types, using the anti-vascular endothelial growth factor (VEGF) monoclonal antibody bevacizumab. Bevacizumab has shown progression free survival benefits in ovarian cancer [2], colorectal cancer [3], non-small cell lung cancer (NSCLC) [4], breast cancer [5], and overall survival benefit in cervical cancer [6]. Preclinical data suggested that anti-angiogenic agents targeting multiple tyrosine kinases might have better anti-tumor activity [7].

Pazopanib is an orally active multi-targeted tyrosine kinase inhibitor of VEGFR-1, 2, and 3, platelet derived growth factor receptor $\alpha$ and $\beta$, fibroblast growth factor receptor 1 and 3 , and c-Kit. It has been approved for the treatment of advanced renal cell carcinoma and advanced soft tissue sarcomas at a dose of $800 \mathrm{mg}$ daily. Tested as a single agent in several phase II studies, pazopanib has also demonstrated efficacy in various types of solid tumors, such as metastatic differentiated thyroid cancers [8], medullary thyroid carcinoma [9], gastroenteropancreatic neuroendocrine tumors [10], and recurrent/metastatic breast cancer treated with up to two prior lines of chemotherapy [11].

Several combinations of pazopanib and chemotherapy have been investigated in clinical trials, using gemcitabine [12], pemetrexed [13, 14], paclitaxel $[15,16]$, paclitaxel and carboplatin [17], paclitaxel and lapatinib [18], docetaxel [19] or ixabepilone [20]. For triple negative breast cancer, where no targeted treatment is approved, recent data favor an activity of platinum salts and a positive impact of anti-angiogenic treatment [5]. Platinum salts are major cytotoxic agents, used in various types of advanced tumors. The feasibility of combining cisplatin with pazopanib was investigated in a phase I, open-label, dose-escalation, and pharmacokinetic (PK) study, to determine the dose-limiting toxicities (DLT) and the maximum tolerated dose (MTD) in patients with recurrent or refractory solid malignancies.

\section{METHODS}

\section{Eligibility Criteria}

Eligible patients were $\geq 18$ year old, had locally advanced, unresectable or metastatic, and histologically confirmed malignant solid tumors, progressing on standard therapy or for 

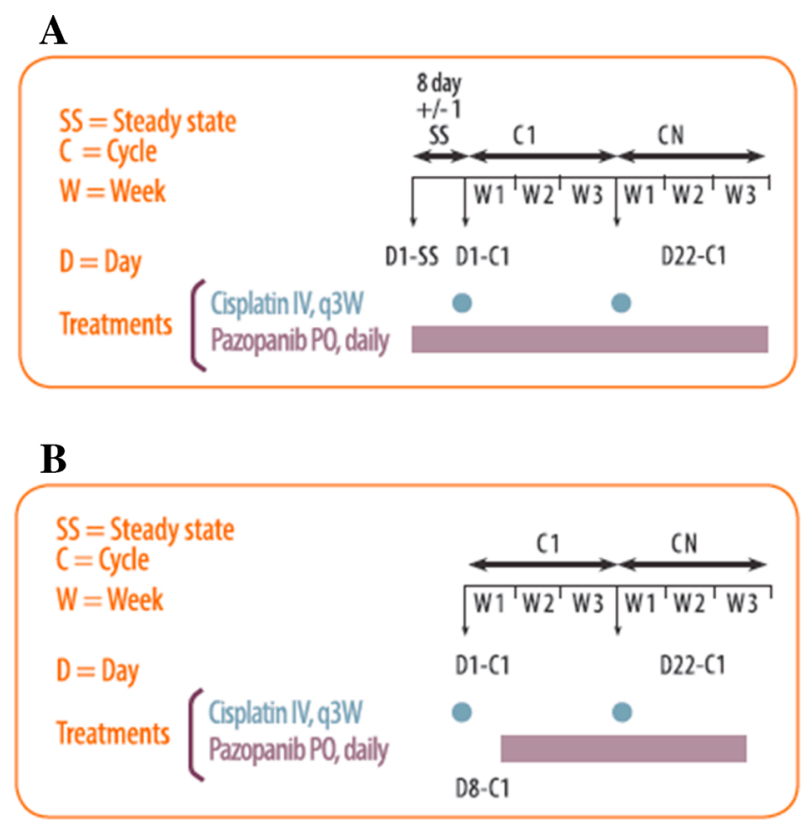

Fig. 1 Treatment regimens and sequences. a Initial sequence. b Reverse sequence: cisplatin administered before pazopanib (cycle 1) and after 14-day pazopanib (cycle 2). $I V$ intravenous, $q 3 \mathrm{~W}$ every 3 weeksand $P O$ per os

whom no standard therapy was available, and candidates for a platinum-based chemotherapy. In the case of breast cancer, patients were eligible if they had received anthracyclines and taxanes for HER2-/HR (hormone receptor)- tumors, anthracyclines, taxanes, and capecitabine for HER2-/HR+ tumors. Other inclusion criteria were Eastern Cooperative Oncology Group performance status of 0 or 1 , adequate hematologic, coagulation and liver function, negative dipstick proteinuria test or positive proteinuria $<1 \mathrm{~g} / 24 \mathrm{H}$, creatinine clearance $>60 \mathrm{ml} / \mathrm{mn}$, and left ventricular ejection fraction (LVEF) $>50$. Patients were excluded if they had HER2 + breast cancers, uncontrolled brain metastases, poorly controlled hypertension, peripheral neuropathy more than grade $(G) 1$, any conditions affecting gastrointestinal functions, high risk of bleeding or anticoagulant therapy, concomitant antineoplastic treatment, previous cisplatin therapy with a cumulated dose $\geq 300 \mathrm{mg} / \mathrm{m}^{2}$, or any concomitant treatment with cytochrome P450 3A4 (CYP3A4) inhibitors or inductors.

\section{Study Design}

This was a phase I, multi-center, open-label dose-finding study, exploring PK interactions and using a $3+3$ enrollment design with a planned expansion at the MTD. In the initial sequence, pazopanib administration (oral morning daily intake in fasted condition) started 8 days before adding cisplatin [intravenously every 3 weeks (q3W)] on day 1 (Fig. 1). Predefined dose levels are shown in Table 1. After 22 patients had been included and the observation of early DLTs, the Data Safety Monitoring Board hypothesized that the unexpected toxicity of the combination might be due to potential interactions of pazopanib on cisplatin PK. Consequently, a reverse sequence (RS) with cisplatin given first (q3W) and pazopanib starting on day 8 (daily) was added after amendment to the protocol, using the 
Table 1 Predefined dose levels for cisplatin and pazopanib

\begin{tabular}{|c|c|c|}
\hline Dose level (DL) & $\begin{array}{l}\text { Cisplatin } \\
\left(\mathrm{mg} / \mathrm{m}^{2} \mathrm{q} 3 \mathrm{~W}\right)\end{array}$ & $\begin{array}{l}\text { Pazopanib } \\
\text { (mg/day) }\end{array}$ \\
\hline DL1 & 75 & 400 \\
\hline DL-1 & 60 & 400 \\
\hline DL2 & 75 & 600 \\
\hline DL3 & 75 & 800 \\
\hline DL4 & 100 & 600 \\
\hline DL-4 & 100 & 400 \\
\hline DL5 & 100 & 800 \\
\hline DL-2 ${ }^{\mathrm{a}}$ & 75 & 200 \\
\hline DL1 reverse sequence ${ }^{a}$ & 75 & 400 \\
\hline
\end{tabular}

${ }^{a}$ DL added during the trial

MTD doses (Fig. 1). The DLT period was 42 days, encompassing cycles 1 and 2. The MTD was defined as the maximal dose regimen for which less than one-third of patients experienced DLT during the DLT period, when at least six evaluable patients had received this regimen [i.e., 0 or 1 patients out of six experienced DLT in this dose level (DL)]. Treatment was pursued until unacceptable toxicity or disease progression.

Due to the high emetogenic potential of cisplatin, all patients received antiemetic comedications: aprepitant $125 \mathrm{mg}$ given orally, methyl prednisolone $80 \mathrm{mg}$ iv, and granisetron $3 \mathrm{mg}$ iv on day 1; aprepitant was continued on days 2 and 3 at $80 \mathrm{mg}$ per day. An interaction between aprepitant and pazopanib was expected. In the case of toxicity, dose modification guidelines were predefined for cisplatin and/or pazopanib.

\section{Assessments}

Screening assessments were performed within 28 days before study entry and included clinical and laboratory examinations, cardiac echography or multi-gated acquisition scan with measurement of LVEF, and imaging [computed tomography (CT) scan and/or magnetic resonance imaging (MRI)]. At the beginning of each cycle, patients underwent an updated history, physical examination, electrocardiogram, and thyroid function, hematology, and chemistry laboratory assessments.

Toxicity was graded according to the National Cancer Institute Common Terminology Criteria for Adverse Events, version 4.03. Toxicity assessments were performed weekly during the first two cycles, and on day 1 of each 21-day cycle thereafter. The determination of DLT was based on tolerance during cycles 1 and 2 and defined as follows: G4 neutropenia lasting more than 7 days, febrile ( $\geq 38.5^{\circ} \mathrm{C}$ ) G3-4 neutropenia, G4 or symptomatic G3 thrombocytopenia, uncontrolled high blood pressure (HBP), despite pazopanib interruption $>2$ weeks, symptomatic HBP, 2 or more symptomatic episodes of HBP despite dose adjustment of pazopanib and antihypertensive treatment, confirmed LVEF decrease (relative decrease $\geq 20 \%$ and LVEF below lower limit of normal), proteinuria $>3 \mathrm{~g} / 24 \mathrm{H}, \mathrm{G} 2$ neuropathy for at least 7 days or G3 neuropathy, any G3-4 non-hematological toxicity, excluding fatigue, anorexia, nausea, vomiting and diarrhea, if considered clinically significant and drug-related by the investigator, inability to administer the planned dose of pazopanib more than 2 weeks in one cycle, delay $\geq 2$ weeks for the second cisplatin administration.

Blood samples were collected for PK evaluation, and a population PK analysis was performed as previously described [21]. Imaging (CT scan or MRI) for efficacy assessment was to be performed every two cycles or sooner if clinically indicated. 


\section{Statistics}

The primary objective of the study was to identify the MTD, based on the rate of DLT occurring during cycles 1 and 2. Patients who progressed before the end of cycle 2 without evidence of DLT were considered not evaluable for the primary objective analysis and replaced during the dose-escalation part. All patients who received at least one dose of either study agent(s) were included in the safety analysis. To be evaluable for DLT, a patient was to be observed for the first two cycles of chemotherapy or to have experienced DLT.

Secondary end points included PK profile of the combination and antitumor activity assessed by RECIST criteria version 1.1. Only descriptive statistics were used, with mean, standard error, median, ranges, quartiles for quantitative variables and counts, and percentages for categorical variables (analyses conducted with SAS version 9.1, SAS Institute Inc, Cary, NC, USA).

\section{Compliance with Ethics Guidelines}

This study was conducted in accordance with the ethical standards of the responsible committee on human experimentation (institutional and national) and with the Helsinki Declaration of 1964, as revised in 2013, in Good Clinical Practices and in European Directive 2001/20/CE regarding the conduct of clinical trials. The study was approved by an independent ethics committee (CPP Ile de France I) and the French National Health Authorities (ANSM). The study was registered in ClinicalTrials.gov (NCT01165385) database. All patients gave written informed consent before participating in the trial.

\section{RESULTS}

In total, 35 patients were included at five sites, from July 2010 to September 2013. Twenty-six were treated according to the initial sequence and 9 according to the reverse sequence (DL1RS), i.e., $75 \mathrm{mg} / \mathrm{m}^{2}$ of cisplatin first and $400 \mathrm{mg}$ of pazopanib. The safety and DLT evaluable populations comprised 34 and 29 patients, respectively. Baseline characteristics of patients, tumors, and prior chemotherapy are summarized in Table 2. Among previous treatments, 32 (91\%) patients had undergone surgery, 20 (57\%) radiation therapy, and 4 (11\%) endocrine therapy. All patients had received prior chemotherapy/targeted therapies except the 4 patients with head and neck cystic adenoid carcinoma.

The patients received a median number of 3 (0-8) cycles with cisplatin and $3(0-28)$ cycles with pazopanib. The cisplatin dose was reduced at least once in 8 patients (23\%) in DL1, 2 and 1RS. Thirteen patients had to discontinue cisplatin (including four in DL2 and 5 in DL1 RS), and seven patients had to discontinue pazopanib (four patients had to discontinue both cisplatin and pazopanib). After discontinuation of cisplatin (either for toxicity or cumulative dose), six patients continued pazopanib alone.

During DL1, 1 DLT occurred [G3 alanine aminotransferase (ALT) elevation] among three evaluable patients. However, G3 anemia and pulmonary embolism occurred in the same slot of patients, and even if they were not accounted as DLT, those toxicities were considered for the management of the dose level. Consequently, in a safe approach, DSMB decided to explore the lower level (DL-1) 
Table 2 Baseline characteristics of patients and tumors

\begin{tabular}{lc}
\hline Variables & Total $(\boldsymbol{n}=\mathbf{3 5})$ \\
\hline Gender (male) & $13(37 \%)$ \\
Age (years) & \\
Median (min-max) & $59(24-72)$ \\
Weight (kg) & \\
Median (min-max) & $67(43-98)$ \\
ECOG & \\
0 & $19(54 \%)$ \\
1 & $16(46 \%)$ \\
Type of malignancy & \\
Ovary & $8(23 \%)$ \\
Sarcoma & $5(14 \%)$ \\
H\&N cystic adenoid carcinoma & $5(14 \%)$ \\
Colorectal & $4(11 \%)$ \\
Breast & $4(11 \%)$ \\
Uveal melanoma & $3(9 \%)$ \\
Endometrial carcinoma & $1(3 \%)$ \\
Liver & $1(3 \%)$ \\
Lung & $1(3 \%)$ \\
Adrenal glands & $1(3 \%)$ \\
Thyroid & $1(3 \%)$ \\
Pancreas & $1(3 \%)$ \\
Prior chemotherapy & \\
Naïve & \\
$\leq 2$ Lumulated dose of prior cisplatin mg/m & \\
Median (min-max) & \\
\hline
\end{tabular}

$H \mho N$ Head and neck, ECOG Eastern Cooperative Oncology Group, $D L$ dose level, $R S$ reverse sequence

${ }^{a}$ All cystic adenoid carcinoma patients

b Five patients treated in DL1, 2 in DL-2, 1 in DL1RS before expanding DL-1 to 3 other patients. No DLT occurred in DL-1.

To better evaluate potential interactions of cisplatin on pazopanib, an additional not preplanned DL-2 was explored. The cisplatin dose was maintained at $75 \mathrm{mg} / \mathrm{m}^{2}$ for efficacy reasons and pazopanib reduced to $200 \mathrm{mg}$ /day to minimize toxicity. No DLT were observed. PK results showed an increase in pazopanib area under curve (AUC) when the drug was taken the days of aprepitant administration. These increases in pazopanib AUC revealed a decrease of pazopanib oral clearance (CL/F with $\mathrm{CL}$ for pazopanib clearance, and $\mathrm{F}$ for oral pazopanib bioavailability).

After DL-1 and DL-2 were considered safe, DL1 was reopened while the protocol was amended with more restrictive inclusion criteria $\quad(\leq 2$ lines of chemotherapy in metastatic setting, no history of anemia or thrombocytopenia $\geq \mathrm{G} 3$ ), to exclude patients with the highest risks of toxicity. DL1RS was then open at MTD to investigate potential interactions of pazopanib on cisplatin PKs that were not considered at the study initiation. DL1RS was considered safe with 1 DLT over 9 evaluable patients. In total, among 29 evaluable patients, 5 experienced at least one DLT: 1 in DL1, 3 in DL2, and 1 in DL1RS (Table 3). Reported DLT was G3 ALT elevations $(n=2)$, G3 pulmonary embolism $(n=2), \quad$ G4 hyponatremia $(\mathrm{n}=1), \mathrm{G} 3$ thrombocytopenia $(n=2)$, and G3 neutropenia $(n=2)$. DL1, i.e., pazopanib $400 \mathrm{mg}$ daily (qd) combined with cisplatin $75 \mathrm{mg} / \mathrm{m}^{2} \mathrm{q} 3 \mathrm{~W}$ was identified as the MTD. Considering the toxicity profile observed in all dose levels, the DSMB decided to not expand the cohort and not explore this combination in a phase II trial in advanced triple negative breast cancer. 
Table 3 Dose levels (DLs) and dose-limiting toxicities (DLTs)

\begin{tabular}{|c|c|c|c|c|c|c|}
\hline DL & Patients & $\begin{array}{l}\text { Cisplatin } \\
\left(\mathbf{m g} / \mathbf{m}^{2}\right)\end{array}$ & $\begin{array}{l}\text { Pazopanib } \\
\text { (mg/day) }\end{array}$ & $\begin{array}{l}\text { DL escalating } \\
\text { order }\end{array}$ & DLT & Description \\
\hline-2 & $3+1 \mathrm{NE}^{a}$ & 75 & 200 & 3 & 0 & \\
\hline-1 & 3 & 60 & 400 & 2 & 0 & \\
\hline 1 & $\begin{array}{l}3+3 \mathrm{NE}^{\mathrm{a}} \\
3+2 \mathrm{NE}^{\mathrm{a}} \\
2\end{array}$ & 75 & 400 & $\begin{array}{l}1 \\
4 \\
6\end{array}$ & 1 & $\begin{array}{l}\text { One patient with G3 ALT elevation and } \\
\text { G3 pulmonary embolism }\end{array}$ \\
\hline 2 & $5+1 N^{a}$ & 75 & 600 & 5 & 3 & $\begin{array}{l}\text { Two patients with G3 } \\
\text { thrombocytopenia }+ \text { G3 neutropenia }{ }^{\mathrm{b}} \\
\text { One patient with G4 hyponatremia }+ \text { G3 } \\
\text { ALT elevation }\end{array}$ \\
\hline $1 \mathrm{RS}$ & 9 & 75 & 400 & 7 & 1 & One patient with G3 pulmonary embolism \\
\hline
\end{tabular}

$G$ grade, $A L T$ alanine aminotransferase, $R S$ reverse sequence

a Non evaluable for DLT

b Resulting in $>2$ weeks delay in C2 cisplatin administration

Most frequent adverse events (all grades) were myelotoxicity (anemia 83\%, neutropenia $73 \%$, and thrombocytopenia $80 \%)$, fatigue (80\%), and hypertension (59\%) (Table 4). G3-4 adverse events were reported in more than $10 \%$ of patients for neutropenia (35\%), hypertension (21\%), fatigue (18\%), anemia (15\%), and thrombocytopenia (15\%).

At the cut-off date, one patient was still treated (desmoid tumor with complete response). Reasons for discontinuation in the remaining 34 patients were unacceptable toxicity in 16 cases (46), tumor progression in 17 patients (50\%) and switch to another antitumor therapy in 1 patient (3\%). Overall, 19 patients died, all from disease progression. The median duration of follow-up after the end of treatment was 9 months (1-20). Four patients had an objective response, including one complete response (one patient with sarcoma) and three partial responses (two patients with ovarian cancer, including one
$>6$ months, and one patient with breast cancer).Twenty patients experienced a stable disease (including one $>6$ months).

PK results have been published elsewhere [21]. Mean [coefficient of variation (CV\%) for inter-individual variability] cisplatin clearance was $10.3 \mathrm{~L} / \mathrm{h} \quad(33 \%)$ and appeared not influenced by pazopanib. However, pazopanib PKs was significantly modified by the cisplatin regimen (most likely due to an interaction of aprepitant on pazopanib metabolism). Mean $(\mathrm{CV} \%)$ of oral pazopanib clearance was $0.66 \mathrm{~L} / \mathrm{h}$ (55\%) at day 0 (before cisplatin administration), $25 \%$ lower at day 1, and 33\% lower at day 2 . This interaction is less likely due to cisplatin than to a competitive inhibition of pazopanib metabolism and efflux by aprepitant, systematically combined with cisplatin. Moreover, the plasma pazopanib exposures observed at day 0 (before cisplatin administration) with a 400-mg dose were similar to those observed at the recommended 
Table 4 Most frequent adverse events across dose-level groups (\% of patients)

\begin{tabular}{|c|c|c|c|c|c|c|c|c|c|c|c|c|}
\hline & \multicolumn{2}{|c|}{ DL-2, $n=4$} & \multicolumn{2}{|c|}{ DL-1, $n=3$} & \multicolumn{2}{|c|}{ DL1, $n=12^{\mathrm{a}}$} & \multicolumn{2}{|c|}{ DL2, $n=6$} & \multicolumn{2}{|c|}{ DL1RS, $n=9$} & \multicolumn{2}{|c|}{ Total, $n=34$} \\
\hline & $\overline{\text { G1-2 }}$ & $\overline{\text { G3-4 }}$ & $\overline{\text { G1-2 }}$ & $\overline{\text { G3-4 }}$ & $\overline{\text { G1-2 }}$ & $\overline{\text { G3-4 }}$ & G1-2 & $\overline{\text { G3-4 }}$ & G1-2 & G3-4 & $\overline{\text { G1-2 }}$ & G3-4 \\
\hline Anemia & 75 & 25 & 100 & 0 & 50 & 25 & 67 & 0 & 78 & 11 & 68 & 15 \\
\hline Neutropenia & 25 & 50 & 67 & 0 & 42 & 17 & 33 & 50 & 33 & 56 & 38 & 35 \\
\hline Thrombocytopenia & 50 & 25 & 67 & 0 & 67 & 9 & 50 & 33 & 44 & 11 & 65 & 15 \\
\hline Anorexia & 50 & 0 & 33 & 0 & 50 & 17 & 33 & 0 & 44 & 11 & 44 & 9 \\
\hline Vomiting & 25 & 0 & 67 & 0 & 50 & 0 & 17 & 17 & 56 & 0 & 44 & 3 \\
\hline Renal failure & 0 & 0 & 0 & 0 & 0 & 0 & 17 & 17 & 22 & 0 & 9 & 3 \\
\hline LVEF decrease & 25 & 0 & 0 & 0 & 0 & 0 & 17 & 17 & 11 & 11 & 9 & 6 \\
\hline Neurotoxicity & 25 & 25 & 33 & 0 & 50 & 0 & 50 & 0 & 78 & 0 & 53 & 3 \\
\hline Dyspnea & 25 & 0 & 0 & 0 & 25 & 9 & 0 & 0 & 33 & 0 & 21 & 3 \\
\hline ALT increase & 25 & 0 & 33 & 0 & 25 & 9 & 33 & 17 & 11 & 0 & 24 & 6 \\
\hline AST increase & 0 & 25 & 33 & 0 & 25 & 17 & 50 & 0 & 11 & 0 & 24 & 9 \\
\hline Hyponatremia & 75 & 0 & 67 & 0 & 33 & 17 & 0 & 17 & 11 & 0 & 29 & 9 \\
\hline Fatigue & 50 & 25 & 67 & 33 & 58 & 17 & 67 & 0 & 67 & 22 & 62 & 18 \\
\hline Hypertension & 0 & 50 & 33 & 0 & 50 & 0 & 33 & 50 & 44 & 22 & 38 & 21 \\
\hline
\end{tabular}

$D L$ dose level, $R S$ reverse sequence, $G$ grade, $L V E F$ left ventricular ejection fraction, $A S T$ aspartate aminotransferase, $A L T$ alanine aminotransferase

a One patient of 13 in DL1 could not be analyzed for toxicity. The safety population included 34 patients

dose of pazopanib in mono-chemotherapy (800 mg) during the first-in-man phase I study [23].

\section{DISCUSSION}

In this study, we assessed the feasibility and the MTD of pazopanib plus cisplatin, a combination that has the potential for providing therapeutic benefits in patients with recurrent/refractory tumors, due to the addition of anti-angiogenic effects to chemotherapy. Main toxicities were myelosuppression, fatigue, and hypertension, consistent with the known toxicity profile of both drugs. It has been shown that the addition of tyrosine kinase inhibitors (TKI) significantly increases toxicity compared to chemotherapy alone, especially myelosuppression, fatigue, skin toxicity, hypertension, and diarrhea [22]. Since no previous study assessed the combination of cisplatin and pazopanib, the starting doses were chosen below those used in monotherapy. In addition, DLT were evaluated over cycles 1 and 2 to observe cumulative toxicities as suggested for targeted agents [23]. The common metabolism of pazopanib and aprepitant was known, and interactions between both drugs were expected. We observed a poor tolerance from the first dose level that corresponded to the MTD, namely, pazopanib $400 \mathrm{mg}$ daily and cisplatin $75 \mathrm{mg} /$ $\mathrm{m}^{2} \mathrm{q} 3 \mathrm{~W}$. DLTs were elevations of liver enzymes, pulmonary embolism, and myelosuppression. 
The safety profile of cisplatin is well known, with renal, gastrointestinal toxicity, and neuropathy. When used as a single agent, pazopanib is considered reasonably tolerated. The first dose-finding study in patients with cancer that yielded the recommendation of the 800-mg daily dose showed limited toxicity, the most frequent being hypertension [24]. In renal cell carcinoma, pazopanib compared to sunitinib appeared to be better tolerated with a few G3 or 4 adverse events [25]. In medullary thyroid carcinoma, G3 or 4 toxicities were infrequent, consisting mostly of fatigue (14\%) or diarrhea (9\%) [9]. In breast cancer, $14 \%$ of patients had G3 or 4 hepatic enzymes increases, $14 \%$ had G3 or 4 neutropenia, and 14\% had G3 hypertension [11].

Several studies have investigated pazopanib and cytotoxic drugs combination. In the phase I dose-escalation study of pazopanib combined with gemcitabine, the MTD could not be determined, since no DLTs were observed at the highest DL, pazopanib $800 \mathrm{mg}$ and gemcitabine $1250 \mathrm{mg} /$ day, i.e., doses used as single agent for each drug [12]. Most frequent adverse events were fatigue, nausea, anorexia, and decreased leucocytes. One-third of patients experienced G4 neutropenia or thrombocytopenia.

The combination with pemetrexed was explored in a phase I study in 25 patients with advanced solid tumors after failure of standard therapy [13]. The MTD was pazopanib $800 \mathrm{mg}$ daily and pemetrexed $500 \mathrm{mg} / \mathrm{m}^{2} \mathrm{q} 3 \mathrm{~W} ; 44 \%$ of patients experienced G4 neutropenia at some point during the study. Two patients out of 20, both with NSCLC, had partial response. These promising results led to perform a phase II trial of pazopanib + pemetrexed compared to cisplatin + pemetrexed in NSCLC [14]. This trial was discontinued prematurely due to unacceptable toxicity in the pazopanib + pemetrexed arm (3 toxic deaths among 61 patients).

Interestingly, pazopanib was safe in combination with continuous infusion of ifosfamide, since doses up to $1000 \mathrm{mg}$ could be administered without major toxicity [26]. However, the same combination with administration of ifosfamide in bolus was poorly tolerated and pazopanib had to be decreased to $200 \mathrm{mg}$.

A phase I trial showed that pazopanib $800 \mathrm{mg}$ daily and paclitaxel $80 \mathrm{mg} / \mathrm{m}^{2}$ at day 1,8 , and 15 in 28-day cycles was feasible [15]. Most frequent G3 or 4 toxicities were liver enzymes increases and neutropenia. The response rate was $19 \%$. Another study assessed the same combination with paclitaxel every 21 days in 26 patients confirmed a manageable safety profile with the same toxicities [16]. The MTD was pazopanib $800 \mathrm{mg}+$ paclitaxel $150 \mathrm{mg} / \mathrm{m}^{2}$.

The 3-drug combination paclitaxel + carboplatin + pazopanib was poorly tolerated (mainly hematologic toxicity) at standard doses in a phase I, dose-finding study [17]. The MTD was pazopanib $200 \mathrm{mg}$, paclitaxel $175 \mathrm{mg} / \mathrm{m}^{2}$, and carboplatin AUC 5. Concurrent administration of pazopanib increased significantly the exposure to paclitaxel and carboplatin, which could explain the greatest toxicity. Among 34 patients, 2 and 4 had complete and partial responses, respectively, (17\% response rate) and another 7 patients presented disease stabilization. The same combination in previously untreated gynecologic tumors had been estimated not feasible due to intolerable toxicity, even at the lowest dose level (pazopanib $400 \mathrm{mg}$, carboplatin AUC 5, paclitaxel $175 \mathrm{mg} / \mathrm{m}^{2}$ every 21 days) [27].

Taken together, these results suggest that the combination of pazopanib with platinum 
derivatives (or salts) might be more toxic than expected at the doses explored. PK interactions may partly explain this finding. We did not find any impact of pazopanib on cisplatin PKs, in contrast to the results obtained by Burrris et al., who showed that carboplatin AUC was higher when administered with pazopanib [17]. However, in our study, we showed an increased exposure to pazopanib with concomitant cisplatin, likely due to the interaction with the CYP3A4 inhibitor aprepitant. The contribution of the decrease in pazopanib oral clearance by aprepitant to the poor tolerance observed during this phase I study should have been limited. Indeed, it concerns only 3 days of treatment among a 21-day cycle since the half-life elimination of aprepitant is around $9 \mathrm{~h}$. The pazopanib concentrations observed at day 0 should be more representative of the daily exposure of the patients. The plasma pazopanib exposures observed at day 0 with a 400-mg dose were similar to those observed at the recommended dose of pazopanib in mono-chemotherapy (800 mg) during the first-in-man phase I study. The observed pazopanib plasma overexposure probably contributed to the poor tolerance encountered during this phase I study.

This study represents the first study revealing a significant PK interaction between aprepitant and a TKI. Usually, aprepitant is not administered in combination with pazopanib, but is suggested as a prolonged treatment of pruritus associated with erlotinib [28]. The current results emphasize the need for further evaluation of the interaction between aprepitant and other TKIs.

The combination of platinum-based chemotherapy and anti-angiogenic multi-target TKIs carries particularly high toxicity, as shown by studies with sunitinib [29-31] or, to a lesser extent with sorafenib
$[32,33]$ mainly due to overlapping of toxicity profile of the drugs. Other strategies such as less toxic chemotherapy or different schedules (weekly, metronomic) or intermittent dosing of pazopanib should be explored. Moreover, a focus on sequential treatment represents an alternative option as demonstrated recently in ovarian carcinoma [34]. Further analysis may identify specific subgroups of patients who may derive benefit of anti-angiogenic drug.

The choice of cisplatin can be questioned, due to its high toxicity. Carboplatin is now preferred in many tumor types, such as ovarian, cervix or breast cancer. However, at the time of writing of this protocol, cisplatin was considered as the leading compound in platinum-based therapies, particularly in breast cancer. Initially, this phase I study was designed to evaluate the combination cisplatin + pazopanib for treatment of advanced triple negative breast cancer in a planned extension cohort, due to preliminary results of cisplatin and sunitinib $[35,36]$ in this indication. The extension phase has been canceled when the combination appeared too toxic to be further explored. However, the authors acknowledge that the $6(17 \%)$ patients who had received prior cisplatin before inclusion in the study may have accounted for the observed toxicity. Finally, the administration of aprepitant, despite predictable interactions with pazopanib, was considered as it is the standard of care of patients receiving cisplatin due to the high emetogenic potential of this cytotoxic drug.

\section{CONCLUSION}

Despite clinical activity observed in four patients, our data show that pazopanib combined with cisplatin may not be tolerable at the dose and regimen tested in this study. 


\section{ACKNOWLEDGMENTS}

This work was funded by GlaxoSmithKline and by the charity Ligue Nationale de Lutte Contre le Cancer. No funding or sponsorship was received for the publication of this article. We thank Anne Visbecq (KP1916) for writing and editorial support in the preparation of this manuscript, and this support was funded by UNICANCER. All named authors meet the International Committee of Medical Journal Editors (ICMJE) criteria for authorship for this manuscript, take responsibility for the integrity of the work as a whole, and have given final approval for the version to be published.

Disclosures. Véronique Diéras discloses consulting fees from Novartis and GSK. Thomas Bachelot discloses grants, consulting fees from Novartis, Roche and AstraZeneca. Nicolas Isambert discloses consulting fees from Pfizer, MSD and support for travel from BMS, Novartis, Lilly, MSD, PharrmaMar. Florence Joly discloses consulting fees from GSK. Marta Jimenez reports grants from GSK, during the conduct of the study. Mario Campone, Christophe Le Tourneau, Philippe Cassier, Emmanuelle Bompas, Pierre Fumoleau, Sabine Noal, Christine Orsini, Diane Charlotte Imbs and Etienne Chatelut have nothing to disclose.

Compliance with Ethical Guidelines. This study was conducted in accordance with the ethical standards of the responsible committee on human experimentation (institutional and national) and with the Helsinki Declaration of 1964, as revised in 2013, in Good Clinical Practices and in European Directive 2001/20/ CE regarding the conduct of clinical trials. The study was approved by an independent ethics committee (CPP Ile de France I) and the French National Health Authorities (ANSM). The study was registered in ClinicalTrials.gov (NCT01165385) database. All patients gave written informed consent before participating in the trial.

Open Access. This article is distributed under the terms of the Creative Commons Attribution-NonCommercial 4.0 International License (http://creativecommons.org/licenses/ by-nc/4.0/), which permits any noncommercial use, distribution, and reproduction in any medium, provided you give appropriate credit to the original author(s) and the source, provide a link to the Creative Commons license, and indicate if changes were made.

\section{REFERENCES}

1. Jain RK. Normalization of tumor vasculature: an emerging concept in antiangiogenic therapy. Science. 2005;307:58-62.

2. Gaitskell K, Martinek I, Bryant A, et al. Angiogenesis inhibitors for the treatment of ovarian cancer. Cochrane Database Syst Rev. 2011; CD007930.

3. Wagner AD, Arnold D, Grothey AA, et al. Anti-angiogenic therapies for metastatic colorectal cancer. Cochrane Database Syst Rev. 2009; CD005392.

4. Sandler A, Gray R, Perry MC, et al. Paclitaxel-carboplatin alone or with bevacizumab for non-small-cell lung cancer. $\mathrm{N}$ Engl J Med. 2006;355:2542-50.

5. Miles DW, Dieras V, Cortes $J$, et al. First-line bevacizumab in combination with chemotherapy for HER2-negative metastatic breast cancer: pooled and subgroup analyses of data from 2447 patients. Ann Oncol. 2013;24:2773-80.

6. Tewari KS, Sill MW, Long HJ 3rd, et al. Improved survival with bevacizumab in advanced cervical cancer. N Engl J Med. 2014;370(8):734-43.

7. Erber R, Thurnher A, Katsen AD, et al. Combined inhibition of VEGF and PDGF signaling enforces tumor vessel regression by interfering with pericyte-mediated endothelial cell survival mechanisms. FASEB J. 2004;18:338-40. 
8. Bible KC, Suman VJ, Molina JR, et al. Efficacy of pazopanib in progressive, radioiodine-refractory, metastatic differentiated thyroid cancers: results of a phase 2 consortium study. Lancet Oncol. 2010;11:962-72.

9. Bible KC, Suman VJ, Molina JR, et al. A multicenter phase 2 trial of pazopanib in metastatic and progressive medullary thyroid carcinoma: MC057H. J Clin Endocrinol Metab. 2014;99:1687-93.

10. Ahn HK, Choi JY, Kim KM, et al. Phase II study of pazopanib monotherapy in metastatic gastroenteropancreatic neuroendocrine tumours. Br J Cancer. 2013;109:1414-9.

11. Taylor SK, Chia S, Dent S, et al. A phase II study of pazopanib in patients with recurrent or metastatic invasive breast carcinoma: a trial of the Princess Margaret Hospital phase II consortium. Oncologist. 2010;15:810-8.

12. Plummer R, Madi A, Jeffels M, et al. A phase I study of pazopanib in combination with gemcitabine in patients with advanced solid tumors. Cancer Chemother Pharmacol. 2013;71:93-101.

13. Infante JR, Novello S, Ma WW, et al. Phase Ib trial of the oral angiogenesis inhibitor pazopanib administered concurrently with pemetrexed in patients with advanced solid tumors. Invest New Drugs. 2013;31:927-36.

14. Scagliotti GV, Felip E, Besse B, et al. An open-label, multicenter, randomized, phase II study of pazopanib in combination with pemetrexed in first-line treatment of patients with advanced-stage non-small-cell lung cancer. J Thorac Oncol. 2013;8:1529-37.

15. Tan AR, Dowlati A, Jones SF, et al. Phase I study of pazopanib in combination with weekly paclitaxel in patients with advanced solid tumors. Oncologist. 2010;15:1253-61.

16. Kendra K, Plummer R, Salgia R, et al. A multicenter phase I study of pazopanib in combination with paclitaxel in first-line treatment of patients with advanced solid tumors. Mol Cancer Ther. 2015;14:461-9.

17. Burris HA 3rd, Dowlati A, Moss RA, et al. Phase I study of pazopanib in combination with paclitaxel and carboplatin given every 21 days in patients with advanced solid tumors. Mol Cancer Ther. 2012;11:1820-8.

18. Tan AR, Dowlati A, Stein MN, et al. Phase I study of weekly paclitaxel in combination with pazopanib and lapatinib in advanced solid malignancies. Br J Cancer. 2014;110:2647-54.
19. Hamberg $\mathrm{P}$, Mathijssen $\mathrm{RH}$, de Bruijn $\mathrm{P}$, et al. Impact of pazopanib on docetaxel exposure: results of a phase I combination study with two different docetaxel schedules. Cancer Chemother Pharmacol. 2015;75:365-71.

20. Ganesan C, Obulareddy SJ, Fischer JH, et al. Phase I study of pazopanib and ixabepilone in patients with solid tumors. Am J Clin Oncol. 2016;39:280-7.

21. Imbs DC, Dieras $\mathrm{V}$, Bachelot $\mathrm{T}$, et al. Pharmacokinetic interaction between pazopanib and cisplatin regimen. Cancer Chemother Pharmacol. 2016;77:385-92.

22. Funakoshi T, Latif A, Galsky MD. Safety and efficacy of addition of VEGFR and EGFR-family oral small-molecule tyrosine kinase inhibitors to cytotoxic chemotherapy in solid cancers: a systematic review and meta-analysis of randomized controlled trials. Cancer Treat Rev. 2014;40:636-47.

23. Paoletti X, Le Tourneau C, Verweij J, et al. Defining dose-limiting toxicity for phase 1 trials of molecularly targeted agents: results of a DLT-TARGETT international survey. Eur J Cancer. 2014;50:2050-6.

24. Hurwitz HI, Dowlati A, Saini S, et al. Phase I trial of pazopanib in patients with advanced cancer. Clin Cancer Res. 2009;15:4220-7.

25. Motzer RJ, Johnson T, Choueiri TK, et al. Hyperbilirubinemia in pazopanib- or sunitinib-treated patients in COMPARZ is associated with UGT1A1 polymorphisms. Ann Oncol. 2013;24:2927-8.

26. Hamberg P, Boers-Sonderen MJ, van der Graaf WT, et al. Pazopanib exposure decreases as a result of an ifosfamide-dependent drug-drug interaction: results of a phase I study. $\mathrm{Br} \mathrm{J}$ Cancer. 2014;110:888-93.

27. du Bois A, Vergote I, Wimberger P, et al. Open-label feasibility study of pazopanib, carboplatin, and paclitaxel in women with newly diagnosed, untreated, gynaecologic tumours: a phase I/II trial of the AGO study group. $\mathrm{Br} \mathrm{J}$ Cancer. 2012;106:629-32.

28. Vincenzi B, Tonini G, Santini D. Aprepitant for erlotinib induced pruritus. $\mathrm{N}$ Engl $\mathrm{J}$ Med. 2010;363:397-8.

29. Heath EI, Blumenschein GR Jr, Cohen RB, et al. Sunitinib in combination with paclitaxel plus carboplatin in patients with advanced solid tumors: phase I study results. Cancer Chemother Pharmacol. 2011;68:703-12. 
30. Geldart T, Chester J, Casbard A, et al. SUCCINCT: an open-label, single-arm, non-randomised, phase 2 trial of gemcitabine and cisplatin chemotherapy in combination with sunitinib as first-line treatment for patients with advanced urothelial carcinoma. Eur Urol. 2015;67:599-602.

31. Blais N, Camidge DR, Jonker DJ, et al. Sunitinib combined with pemetrexed and carboplatin in patients with advanced solid malignancies-results of a phase I dose-escalation study. Invest New Drugs. 2013;31:1487-98.

32. Polcher M, Eckhardt M, Coch C, et al. Sorafenib in combination with carboplatin and paclitaxel as neoadjuvant chemotherapy in patients with advanced ovarian cancer. Cancer Chemother Pharmacol. 2010;66:203-7.

33. Lee JK, Capanu M, O'Reilly EM, et al. A phase II study of gemcitabine and cisplatin plus sorafenib in patients with advanced biliary adenocarcinomas. $\mathrm{Br}$ J Cancer. 2013;109:915-9.

34. du Bois A, Floquet A, Kim JW, et al. Incorporation of pazopanib in maintenance therapy of ovarian cancer. J Clin Oncol. 2014;32:3374-82.

35. Burstein HJ, Elias AD, Rugo HS, et al. Phase II study of sunitinib malate, an oral multitargeted tyrosine kinase inhibitor, in patients with metastatic breast cancer previously treated with an anthracycline and a taxane. J Clin Oncol. 2008;26:1810-6.

36. Isakoff SJ, Mayer EL, He L, et al. TBCRC009: a multicenter phase II clinical trial of platinum monotherapy with biomarker assessment in metastatic triple-negative breast cancer. J Clin Oncol. 2015;33:1902-9. 